\title{
Estrategia Etnoeducativa sobre cuidado del medio ambiente apoyada en saberes ancestrales de etnia Emberá Katío
} Ethno-educational strategy on caring for the environment supported by ancestral knowledge of the Embera Katío ethnic group

\section{Elizabeth Cantero Galarcio ${ }^{1}$}

Facultad de Educación y Ciencias Humanas, Universidad de Córdoba, Montería, Colombia

\section{Elías Enrique Hernández Hernández ${ }^{2}$}

Facultad de Educación y Ciencias Humanas, Universidad de Córdoba, Montería, Colombia

\section{Luis Carlos Pacheco Lora ${ }^{3}$}

Facultad de Educación y Ciencias Humanas, Universidad de Córdoba, Grupo de Investigación CYMTED-L, Montería, Colombia

1 Bióloga, Maestrante de Didáctica de las Ciencias Naturales Universidad de Córdoba, Docente del sector público Institución Educativa los Volcanes de Tierralta - Córdoba, ecanterogalarcio@correo.unicordoba.edu.co, teléfono 3207323064, https://orcid.org/0000-0001-9522-3562

$2 \quad$ Licenciado en Ciencias Naturales y Educación Ambiental, Maestrante de Didáctica de las Ciencias Naturales Universidad de Córdoba, Docente del sector público Institución Educativa los Morales de Tierralta - Córdoba, ehernandezhernandez@correo.unicordoba.edu.co,teléfono 3116528024, https://orcid.org/0000-0001-8819-3051

3 Doctor en Psicología, Docente asociado, Universidad de Córdoba, Grupo de Investigación CYMTED-L, Coordinador Semillero de investigación SENTIA, programa Licenciatura en Educación Infantil, Icpacheco@correo. unicordoba.edu.co, teléfono 3156992794, https://orcid. org/0000-0003-0676-9120

\section{RESUMEN}

El actual artículo tiene como propósito presentar el diseño de una estrategia etnoeducativa desde los saberes ancestrales de la etnia Emberá Katío sobre el cuidado del medio ambiente para el área de Ciencias Naturales y Educación Ambiental del grado 5 básica primaria, sede Volador de la Institución Educativa Los Morales de Tierralta, Córdoba. A partir del paradigma interpretativo - hermenéutico, un diseño etnográfico con 
enfoque cualitativo y con la aplicación de técnicas de observación, entrevista y grupo focal, se indaga una muestra de 14 estudiantes indígenas, un docente occidental, dos docentes Emberá Katío y cinco miembros de la comunidad Doza (el Jaibaná, Alguacil, Hierbatero, Cazador y Anciano). Se obtiene como resultado una estrategia etnoeducativa con cinco talleres que hacen énfasis en las categorías que emergieron de la caracterización e interpretación de la información: Territorio, Plantas Medicinales, Alimentación, Chagra y Memorias. En conclusión, se logra una estrategia que incorpora una secuencia didáctica de talleres, con perspectiva de interculturalidad, en los que se propicia el diálogo de saberes entre las Ciencias Naturales y las Cosmovisiones Indígenas acerca de la naturaleza y el medio ambiente. Se propone que los talleres se implementen mediante el método reflexión- acción-participación (rap) en el área de ciencias naturales y educación ambiental de la básica primaria.

PALABRAS CLAVES: Etnia Emberá Katío, Saberes ancestrales, Estrategia etnoeducativa, Ciencias naturales, Educación ambiental

\section{ABSTRACT}

The current article aims to present the design of an ethno-educational strategy based on the ancestral knowledge of the Emberá Katío ethnic group on caring for the environment for the area of Natural Sciences and Environmental Education of grade 5 primary school, Volador headquarters of the Educational Institution Los Morales de Tierralta, Córdoba. Starting from the interpretive-hermeneutical paradigm, an ethnographic design with a qualitative approach and with the application of observation, interview and focus group techniques, a sample of 14 indigenous students, a Western teacher, two Emberá Katío teachers and five members of the Doza community (the Jaibaná, Alguacil, Herbatero, Hunter and Elder). The result is an ethno-educational strategy with five workshops that emphasize the categories that emerged from the characterization and interpretation of the information: Territory, Medicinal Plants, Food, Chagra and Memories. In conclusion, a strategy is achieved that incorporates a didactic sequence of workshops, with an intercultural perspective, in which the dialogue of knowledge between Natural Sciences and Indigenous Worldviews about nature and the environment is fostered. It is proposed that the workshops be implemented through the reflection-action-participation (rap) method in the area of natural sciences and environmental education of elementary school.

KEY WORDS: Emberá Katío Ethnicity, Ancestral knowledge, Ethno-educational strategy, Natural sciences, Environmental Education

\section{INTRODUCCIÓN}

El presente artículo se inscribe en el proyecto de investigación "Estrategia etnoeducativa desde los saberes ancestrales de la etnia Emberá Katío sobre el cuidado del medio ambiente", que plantea el cuestionamiento relativo a cómo los saberes ancestrales de la etnia Emberá Katío sobre el cuidado del medio pueden contribuir al diseño de una estrategia etnoeducativa para el área de Ciencias Naturales y Educación Ambiental del grado 5 de básica primaria, sede Volador de la Institución Educativa los Morales de Tierralta, Córdoba. Como parte del estudio citado, este escrito tiene como propósito dar a conocer el diseño de la estrategia etnoeducativa desde los saberes ancestrales de la etnia Emberá Katío sobre el cuidado del medio ambiente. La estrategia incorpora una secuencia didáctica de talleres, en cuya perspectiva de interculturalidad, se propicia el diálogo de saberes entre las Ciencias Naturales y las Cosmovisiones Indígenas acerca de la naturaleza y el medio ambiente.

Para comprender la problemática y las necesidades que justifican este estudio, es preciso caracterizar inicialmente la población y el 
contexto objeto de análisis. En el departamento de Córdoba se destaca la presencia de dos etnias indígenas: Zenú y Emberá. Los Emberá Katío del departamento de Córdoba equivalen al $12,1 \%$ de la población nacional de esta etnia y se ubican en los municipios de Puerto Libertador, Montelíbano, San José de Uré y Tierralta, siendo este último el lugar donde se concentra la mayor parte de la población departamental. De los indígenas registrados en el departamento de Córdoba, el $82 \%$ fueron identificados como analfabeta, y un alto porcentaje de esta población son niños en estado de vulnerabilidad por conflicto armado u otras problemáticas sociales. (DANE, 2019).

El Plan de Desarrollo 2016-2019 de la Alcaldía de Tierralta registra que la Institución Educativa Los Morales tiene una población de 1013 estudiantes y 47 docentes, distribuidos en tres sedes: el establecimiento Educativo Los Morales, la Escuela Rural Los Arapios y el Centro Educativo Volador. Este último incluye 132 estudiantes y 6 docentes. (p. 132).

A esta Institución Educativa, como a otras del Municipio de Tierralta, asisten niños y niñas del Resguardo Emberá Katío del Alto Sinú, que está integrado por 28 comunidades indígenas: 26 dentro del resguardo; otras 3 ubicadas por fuera en las poblaciones de Crucito, Antadó y Zarandó (cerca de los ríos Sinú, Verde y Esmeralda); y otras 10 que se encuentran más separadas del resguardo, con grupos poblacionales ubicados en veredas y barrios del casco urbano. (Plan de Desarrollo 2016-2019. Alcaldía de Tierralta, p. 41).

En lo que respecta a la estructura social y educativa de la comunidad Emberá del alto Sinú, la familia -conformada por padre, madre e hijos-, es la unidad básica productora y educativa. En el resguardo, la comunidad lleva a cabo el proceso de educación propia hasta quinto de primaria, la educación infantil contempla tres ejes fundamentales, matemáticas, territorio $\mathrm{y}$ cosmovisión, impartidas por educadores de la comunidad Emberá que se preparan fuera del resguardo para impartir las clases dentro de la comunidad (Castillo, 2015).

Según la cosmovisión de los Emberá Katío, sus creencias y su pensamiento colectivo sobre el mundo y la naturaleza, destacan que todo los que habita sobre la tierra tiene "espíritu". Dentro de esta convicción ancestral concurren los lugares prohibidos, encantados y comunales: los lugares prohibidos son aquellos donde no se pueden llevar a cabo actividades de pesca, caza, o corte de madera, en estos lugares habita el creador, sitios como los cementerios, ríos, montañas, lagos, lagunas, quebradas, sitios de origen y manglares se consideran prohibidos; los sitios encantados son zonas donde no se puede entrar sin pedir permiso de los seres espirituales mediante rituales de limpieza y armonización, entre ellos resalta el Río San Juan, Río Atrato, Río San Jorge, lagos, lagunas, montañas, mar, quebradas, bosque entre otros; los sitios comunales son aquellos donde se realizan las actividades cotidianas, como los tambos, áreas de pesca o caza (Vallejo \& Majore, 2018).

Por otra parte, la tradición oral es el medio de trasmisión del conocimiento para los Emberá. De modo oral, los niños aprenden la lengua materna hasta los cuatro años de edad y al llegar a la escuela adoptan el español como segunda lengua. Sin embargo, no todos los miembros de la etnia han logrado acceder a la educación propia y es baja la tasa de alfabetización de la comunidad; en el resguardo del alto Sinú, en especial la comunidad Doza de donde son parte la mayoría de estudiantes de la sede educativa Volador, se habla la lengua nativa Emberá bédea y una fracción menor habla castellano. Aunque no existen cifras concretas sobre la tasa de analfabetismo, es de interés general que los miembros de la comunidad accedan a programas de educación (Vallejo y Majore, 2018). 
Los Emberá del alto Sinú en su historia reciente han sido víctimas del conflicto armado, del despojo de tierras y del desplazamiento forzado, situaciones intensificadas por los grupos al margen de la ley y la construcción de la represa del proyecto de Hidroeléctrica Urrá, que requirió la inundación de una importante área del territorio ocupado. Estas tensiones produjeron unas dinámicas masivas de desplazamiento en las comunidades de la región y el desarraigo en una colectividad donde la tierra es la base de su cosmovisión y cultura. (Aceros, 2019).

Este desplazamiento continuo y prolongado de la población Emberá, desde sus zonas de residencia hasta los centros poblados de los municipios de influencia e incluso a las capitales departamentales, ha apremiado en la manera como los niños y jóvenes Emberá afrontan su educación. Es así como los niños Emberá se enfrentan a un proceso de occidentalización de su cultura, a la pérdida sistemática de la identidad y su sentido de territorio. (Medina \& Cantero, 2018). En este orden de ideas, las consecuencias son acentuadas puesto que la mayoría de las instituciones educativas del entorno no implementan el enfoque diferencial requerido por esta población. Los niños Emberá en edad escolar víctimas del desplazamiento, se ven obligados a interiorizar una cultura totalmente ajena, sin las bases necesarias para afrontar la pérdida de sus usos, costumbres ancestrales y el desarraigo territorial. De esta forma, el proceso de escolarización se empeña en los aspectos científicos emplazados en los currículos oficiales, pero desconoce los saberes y procesos culturales de la comunidad Emberá.

Esto que sucede con los Emberá, es una muestra de que la educación en Colombia tiene dificultades para satisfacer las necesidades de las comunidades indígenas y equilibrar los programas educativos con los requerimientos culturales de cada comunidad, los niños indígenas en condición de vulnerabilidad se ven obligados a adaptarse a un sistema educativo no acorde con sus necesidades.

Particularmente, los niños pertenecientes a la etnia Emberá Katío residentes en la vereda Voladordelmunicipiode Tierralta, enfrentanserios inconvenientes de aprendizaje en diferentes áreas, incluyendo ciencias naturales. Víctimas del desplazamiento forzado, deben integrarse a la comunidad escolar a edades inusuales para el grado a cursar, sin las bases necesarias o rutina escolar; a los niños se les dificulta adquirir nuevos conocimientos y la situación se agrava, cuando la institución educativa a la que están inscritos no cuenta con las herramientas necesarias para brindarles la educación propia a la que tienen derecho. Es así como los niños Emberá adquieren conocimientos hegemónicos en el área de ciencias y se desconocen sus saberes ancestrales, lo que genera un sesgo en el aprendizaje de esta población.

Por otra parte, las observaciones realizadas por los investigadores en el contexto indican que, las dificultades de adaptación a las dinámicas escolares favorece el fenómeno de la deserción en algunos de ellos. Así mismo, en distintas reuniones institucionales y en los procesos de autoevaluación, sale a colación fuertemente la falta de incorporación al currículo de ejes temáticos, de metodologías etnoeducativas y también la falta de capacitación a los docentes que ejercen su función en contextos interculturales.

Los elementos del contexto aludido y las problemáticas evidenciadas, ponen de presente la pertinencia de la investigación en cuanto a la exclusión didáctica/pedagógica que enfrentan los niños y niñas de la etnia Emberá Katío de la sede educativa Volador, problemática que fue corroborada al determinar las características de las prácticas docentes en clase de Ciencias Naturales y Educación Ambiental, en el grado 5 de básica primaria. Las observaciones permiten inferir que en las prácticas docentes 
no se desarrollan estrategias etnoeducativas, lo que es perjudicial en contextos interculturales; también, al examinar el plan de clases, no hay ejes temáticos ni metodológicos acorde a la cultura indígena que se maneja.

La característica principal de esta problemática es la desintegración de las prácticas culturales y tradicionales de los niños y niñas indígenas, como también la pérdida de los saberes ancestrales, a esto se suma el racismo y la exclusión por parte de algunos de sus compañeros, trayendo como consecuencia la desmotivación, el mal rendimiento académico y la deserción de los niños y niñas indígenas.

Asimismo, el estudio es relevante en lo teórico pues acude a un acopio de antecedentes, conceptos, teorías, para darle rigurosidad científica, sirviendo de referentes para influir positivamente en los interrogantes de la temática de estudio. En lo práctico, es importante ya que valida técnicas de recolección de información, como también una estrategia etnoeducativa construida en base a los saberes ancestrales de la etnia Emberá Katío sobre el cuidado del medio ambiente exclusiva para el área de Ciencias Naturales y Educación Ambiental de básica primaria, buscando convertir esta investigación educativa en un recurso de apoyo que facilite al docente acciones etnoeducativas en contexto escolar intercultural, comprometidas con el mejoramiento de los fines educativos.

En suma, la persistencia de dificultades señaladas: la falta de circulación de los conocimientos ancestrales, la carencia de un currículo propio, la falta de participación de la comunidad indígena en la escuela, la no inclusión de estrategias etnoeducativas que visibilicen los saberes ancestrales indígenas, como también la falta de gestión de las directivas para la transformación del currículo hacia una escuela intercultural, donde los docentes estén direccionados a prácticas educativas dialogantes entre los saberes ancestrales indígenas y los de las comunidades no indígenas, plantea el propósito investigativo de diseñar una estrategia etnoeducativa para el área de Ciencias Naturales y Educación Ambiental desde los saberes ancestrales de la cultura Emberá Katío sobre el cuidado del medio ambiente, para posibilitar el rescate de aspectos relevantes de la educación tradicional, en especial la educación ambiental, e incorporarlos a la estructura curricular.

\section{ANTECEDENTES Y MARCO TEÓRICO}

Respecto de estudios previos en el contexto internacional, se especifican los hallazgos con comunidades indígenas de Chile y México de Quilaqueo, Sartorello y Torres (2020) y en Ecuador, Solís (2019); en relación a los antecedentes a nivel nacional, en la Guajira, un estudio de Melo (2019). En términos generales, los antecedentes referidos aportan a la necesidad de diseñar estrategias etnoeducativas que apunten a la incorporación y articulación de la escuela occidental con los saberes ancestrales de las comunidades indígenas, en este caso, los saberes sobre el medio ambiente de la etnia Emberá Katío, con el propósito de que esos saberes etnoeducativos emerjan en la praxis escolar y logren transformaciones en el plan de área y en el proceso de enseñanza/aprendizaje de las Ciencias Naturales y Educación Ambiental. (Para mayor información confrontar los referentes bibliográficos citados).

En ese orden de ideas, es preciso que la estrategia que se propone se encuadre en metodologías adecuadas para su desarrollo basadas en las teorías del constructivismo. Al revisar antecedentes como (Fresneda, 2019; Muñoz, 2018; Peña, 2016; SED de Bogotá, 2014) en este tipo de estrategias etnoeducativas en ambientes multiculturales se recomienda el método basado en la Reflexión - Acción - Participación (RAP), que "concibe el conocimiento como una construcción colectiva y otorga un importante papel a los saberes populares" (SED, 2014, p. 31). Desde 
el escenario educativo este método pone esos saberes, experiencias, habilidades en función de una transformación social, que involucra la acción (social, ambiental, cultural, religiosa, económica, entre otras) de los individuos y las comunidades.

En cuanto al marco teórico y conceptual, el presente estudio asume referentes como el de Saberes Ancestrales Indígenas. González define que los saberes ancestrales constituyen

un conjunto o acumulación de conocimientos prácticos y creencias, obtenidos y desarrollados a través de la observación y experimentación de las poblaciones o sociedades autóctonas con los elementos y condiciones específicos de su hábitat o entorno, para garantizar la supervivencia y satisfacer las necesidades de su comunidad. Estos conocimientos versan sobre la naturaleza y sus procesos, sobre las relaciones entre sus componentes (animales, plantas, y ecosistemas) y los seres humanos, y sobre las relaciones de estos entre sí (relaciones sociales, espirituales y políticas). $(2015$, p.8)

Muñoz, Giraldo y López (2019), identifican cuatro elementos claves que apuntan a reivindicar la significación de los saberes ancestrales: los pueblos indígenas, el territorio, el vínculo existente entre estos dos y los derechos inmanentes a estos saberes ancestrales. Entre estos elementos existe una relación muy estrecha. Justamente, el vínculo entre las comunidades indígenas y sus territorios está dado en gran medida por sus cosmovisiones y la herencia ancestral del territorio, el cual deben respetar, salvaguardando su equilibrio. Por otra parte, la idea de territorio es concebida como un espacio o lugar donde la comunidad despliega sus actividades sociales, económicas y culturales. Además, desde la cosmogonía indígena, el territorio se asocia con lo sagrado y lo ancestral.

Detrás de esta cosmovisión indígena, no solo del territorio sino también de sus actividades sociales, económicas y culturales, existe un sistema de conocimiento ecológico indígena indiscutiblemente opuesto al sistema de conocimiento científico. En este sentido, Muñoz, et.al. (2019), destacan características encontradas: mientras en el sistema de conocimiento ecológico indígena prima lo cualitativo, en el sistema de conocimiento científico, lo cuantitativo; en la perspectiva indígena prevalece el conocimiento integral, holístico, fundado en creencias, tradiciones y un profundo animismo, entre tanto la mirada del sistema científico es racional-analítico y abstracto; por otra parte, en los pueblos indígenas la forma de transmitir el conocimiento es de forma oral y a través de la experiencia compartida, mientras en el modelo científico el conocimiento se revela de forma escrita y prosiguiendo un método y un sistema de validación por una comunidad académica.

Otros conceptos de virtual importancia para los fines de este estudio corresponde a la Etnoeducación y el Diálogo de Saberes. Desde ciertos análisis se ha manifestado la importancia de la etnoeducación en la recuperación de saberes ancestrales para hacerlos partícipes en el currículo. Bajo esta perspectiva, la etnoeducación contempla los procesos de rescate, sistematización y resignificación de los conocimientos y experiencias de las comunidades minoritarias, de tal manera que a partir de estas raíces se pueda alimentar un diálogo entre culturas, cada una con sus conocimientos, valores $\mathrm{y}$, no obstante las tensiones que se puedan generar, se persigue como finalidad ampliar la comprensión del quehacer de los saberes ancestrales en la educación. (Pérez \& Mendoza, 2018). 
Este diálogo entre culturas sugiere la promoción desde los proyectos educativos, desde las áreas de conocimiento y desde las aulas, hacia una apertura al cambio tanto en lo educativo como en lo personal, para desplegar el diálogo entre saberes y entre visiones diferentes del mundo con el propósito de suscitar dicho pensamiento en los estudiantes. Es a partir de este diálogo que González sostiene que la escuela no debe ser solo para transmitir conocimiento sino también "un espacio donde se pueda reafirmar la identidad cultural de los niños a través de su contacto con niños de otras culturas, en un ambiente de respeto e igualdad..." (2010, p.81).

Respecto de la Didáctica de las Ciencias Naturales, Aguado y Campo (2018) ponen de presente los distintos modelos didácticos que han existido a lo largo de su reciente historia de aproximadamente unos 30 años. En efecto, los primero cambios curriculares de Ciencias Naturales, en la década del 60, apuntaba a superar los enfoques tradicionales de "enseñanza por transmisión" (Soler, Cárdenas \& Hernández-Pina, 2018), donde el docente es el poseedor del conocimiento, expositor de conceptos y evaluador de memoria; la única actividad esperada de los alumnos era la asimilación de los contenidos impartidos por el maestro (Leymonié, 2009, p. 28). Durante los años 70 , se esparció el método de enseñanza de las ciencias basados en la "enseñanza por descubrimiento autónomo" (Bruner, 1961), lo que permitió integrar las ciencias, y los estudiantes a desarrollar habilidades como la curiosidad, imaginación, la capacidad de crear y la aplicación del pensamiento crítico y reflexivo en distintas situaciones. Con el inicio de la década de los 80 , la didáctica de las Ciencias Naturales adoptó nuevos referentes epistemológicos del aprendizaje; desde la psicología cognitiva se le da importancia a los concepciones previas (Ausubel, 1998), a estudiar cómo los niños y niñas aprenden ciencias, tratando de entender los procesos y cómo robustecen o reestructuran los conocimientos que ya poseen (incorporación de nuevos conceptos). Aguado y Campo (2018) apoyados en referentes de Ausubel (1998), consideran que las influencias de la epistemología y de la psicología del aprendizaje "sobre la enseñanza de las ciencias provocan una marcada tendencia a investigar sobre las concepciones que los alumnos tienen acerca de los fenómenos naturales antes de recibir una enseñanza científica" (p. 74), las Ilamadas ideas previas o preconceptos, que son concepciones espontáneas. Por otra parte Pozo y Gómez (1998), destacan la tendencia epistemológica y psicológica constructivista en la que el maestro ha tenido cambios en su metodología de trabajo, que incluye transformaciones didácticas en el tratamiento del contenido, en las metodologías de enseñanza, en la forma de evaluar, a la vez que los alumnos también transforman su actuar metodológico, pasan de una enseñanza pasiva a una activa, donde experimentan, solucionan problemas, interactúan directamente con la naturaleza y no se les desconoce sus presaberes, serían los aspectos más relevantes de este nuevo enfoque metodológico.

Atendiendo a los fines del presente estudio, resulta relevante la concepción constructivista, en cuanto a que este modelo en un contexto intercultural sugiere tener en cuenta los conocimientos previos de cada estudiante y su contexto, en el caso de los niños y niñas Emberá Katío es indispensable que el docente cuente con la metodología, conocimiento y herramientas necesarias para hacer del aprendizaje de los estudiantes algo significativo. Implica, entonces, un claro conocimiento de los saberes ancestrales, cultura, ritos, leyendas, entre otros que faciliten el proceso de enseñanza y aprendizaje en la etnia que está involucrada y además poseer la destreza de interrelacionar y lograr un diálogo de saberes, conocimientos y experiencias entre distintas culturas que educa. En este sentido, es prevaleciente reconocer las características y los conocimientos previos con 
los que los alumnos llegan al aula, los deben ser aprovechados para la construcción del nuevo conocimiento.

\section{METODOLOGÍA}

La investigación se sustenta en el paradigma Interpretativo - Hermenéutico que "parte de reconocer la diferencia existente entre los fenómenos sociales y naturales, buscando la mayor complejidad y el carácter inacabado de los primeros, que están siempre condicionados por la participación del hombre" (Barrero, Bohórquez y Mejía 2011, p. 106). En cuanto al enfoque, la investigación se encuadra en el enfoque cualitativo. Al respecto, Hernández, Fernández y Baptista (2014) señalan que el enfoque cualitativo permite describir, comprender e interpretar los fenómenos, a través de la información y significados producidos por las experiencias de los participantes, En este punto es donde se enlaza al presente estudio con la praxis educativa en el escenario de las ciencias naturales, ya que como parte de la investigación en conjunto, se caracterizaron los saberes ancestrales, permitiéndole al investigador reflexionar y tener soporte para diseñar la estrategia etnoeducativa basada en esos mismos saberes para su inclusión en el área de Ciencias Naturales y Educación Ambiental de grado quinto. El tipo de investigación es etnográfica. Según Hernández et al. "pretende explorar, examinar y entender sistemas sociales (grupos, comunidades, culturas y sociedades), así como producir interpretaciones profundas y significados culturales, desde la perspectiva o punto de vista de los participantes o nativos" (2014, p. 482), Con este diseño, a partir de la comprensión que se logra de la cultura ancestral, se propone el diseño de la estrategia etnoeducativa desde los saberes ancestrales de la etnia Emberá Katío sobre el cuidado del medio ambiente.

La población son 133 estudiantes de la sede educativa Volador, 7 docentes y 219 miembros de la comunidad Doza de la etnia Emberá Katío para un total de 359 personas. La muestra se eligió mediante criterios de selección no probabilístico, por conveniencia y con base en juicios puntuales y pertinentes para la investigación. Fueron seleccionados 1 docente de básica primaria de la sede Volador de la Institución Educativa los Morales TierraltaCórdoba, 14 estudiantes Emberá del grado 5 de la mencionada sede, 2 docentes que conforman el equipo de educación en la comunidad DOZA de indígenas Emberá Katío, 5 integrantes de la comunidad DOZA de indígenas Emberá Katío (Jaibaná, el Alguacil, el Hierbatero, un Cazador y un miembro de los más ancianos).

La categoría de análisis para el caso de este artículo es Estrategia Etnoeducativa, con la que se pretende la estructuración de una propuesta que integre los saberes ancestrales al plan educativo de Ciencias Naturales y Educación Ambiental. De igual manera, las técnicas de recolección de información utilizadas en esta investigación corresponden a la observación no participante, la entrevista y el grupo focal, mediante las que se obtuvo información apreciable en cuanto al conocimiento y el cuidado medio ambiental que se han dado de generación en generación en la comunidad indígena. Las fases del estudio son: Diagnóstico, Caracterización y Diseño de la estrategia. La fase que este artículo puntualiza es la del diseño de la estrategia etnoeducativa desde los saberes ancestrales de la etnia Emberá Katío sobre el cuidado del medio ambiente para el área de Ciencias Naturales del grado 5 básica primaria, que seguidamente se despliega en los resultados.

\section{RESULTADOS}

A partir del proceso investigativo desplegado, con la información recolectada y con los datos procesados y triangulados a partir de la observación del contexto, de las entrevistas a los docentes y del grupo focal con integrantes 
de la comunidad Doza etnia Emberá Katío más representativos como el Jaibaná, Alguacil, Hierbatero, Cazador y Anciano, se identificaron puntos de encuentro entre sus respuestas según las dimensiones (Conocimiento, Prácticas y Rituales) de la categoría Saberes ancestrales sobre el cuidado del medio ambiente. En síntesis, se reconocen cinco categorías emergentes Territorio, Plantas medicinales, Alimentación, Chagra y Memorias, las cuales mediante un proceso pedagógico dirigido al desarrollo de competencias en Ciencias Naturales y Educación Ambiental, se pueden incluir como estrategias didácticas acordes al contexto intercultural donde se apliquen.

Estas categorías brindan una ruta temática del componente biológico/ambiental para abordar en el contexto educativo intercultural con la etnoeducación, pero se necesita también un método que brinde la mejor ruta para desarrollar la estrategia etnoeducativa, para lo cual se sugiere el método basado en la ReflexiónAcción-Participación, como quedó sustentado en el marco teórico. A continuación, en la Tabla 1 se presenta la propuesta:

Tabla 1. Propuesta etnoeducativa

\section{Propuesta etnoeducativa de integración de saberes ancestrales de la etnia Emberá Katío sobre el cuidado del medio ambiente al plan educativo de Ciencias Naturales y Educación Ambiental del grado 5 básica primaria}

Introducción: En la presente propuesta se exponen 5 talleres que se enmarcan en los saberes ancestrales de la etnia Emberá Katío sobre el cuidado del medio ambiente con el propósito de ser integrados al plan de área de Ciencias Naturales y Educación Ambiental del grado 5 de básica primaria. Los talleres son productos de la información obtenida en el análisis del trabajo de campo en la comunidad Doza de donde emergieron 5 categorías: Territorio, Plantas medicinales, Alimentación, Chagra y Memorias, las cuales son abordadas conceptualmente en esta propuesta desde lo evidenciado.

Justificación: Por medio de la autoevaluación institucional del año 2018 y una observación a la praxis educativa en clases de Ciencias Naturales y Educación Ambiental en la sede Volador de la Institución Educativa los Morales donde se encuentran niños y niñas indígenas de la etnia Emberá Katío se evidencio unas prácticas excluyentes en relación a la metodología y temática, ya que, son $100 \%$ actuaciones del contexto no indígena u occidentales como las llaman los Emberá, lo que trae como consecuencias la desmotivación por el estudio de los niños y niñas indígenas. Es por ello, la importancia de la presente propuesta, ya que, desde el análisis de los saberes ancestrales de la etnia Emberá Katío sobre el cuidado del medio ambiente se pretende favorecer la inclusión de nuevas prácticas pedagógicas que reconozcan los saberes ancestrales sobre el medio ambiente sus planes, metodologías o propuestas.

\section{Objetivos:}

- Promover la participación activa de los estudiantes en los procesos de enseñanza-aprendizaje a partir del reconocimiento de los saberes ancestrales sobre el medio ambiente de la etnia Emberá Katío. 


\section{Perspectiva desde los saberes ancestrales sobre el cuidado del Medio}

\section{Ambiente}

Conocimiento: la cosmovisión de los indígenas Emberá Katío sobre el medio ambiente se traduce en el territorio, el cual protegen y respeta por su carácter sagrado ya que les fue otorgado por Caragabi que es el Dios en que creen para la obtención del alimento, agua y memoria de sus distintas prácticas. De igual forma son conocedores de las distintas explotaciones que le dan a este territorio desde aprendizajes ancestrales.

Prácticas: Las prácticas de conservación del medio ambiente son amigables y de cierto modo sostenibles, aunque ancestralmente para obtener la carne con la que se alimentan realizan prácticas de caza de animales silvestres actividad que conservan al día de hoy, lo cual visto desde la cultura occidental o no indígena es contribuir a la desaparición o extinción de las especies, en todo caso son prácticas que no son regulares y escogen animales adultos, esto hace parte de su cultura y raíces. De igual forma, conservan costumbres ancestrales como la utilización de plantas medicinales, que es muy relevante ya que confían en sus poderes más que la medicina occidental o no indígena, también practican algunos ritos pero esta actividad es exclusiva del Jaibaná que es un miembro considerado sagrado y con poderes espirituales, sanativos y de protección. En todo el análisis, llama la atención de pequeños cultivos cerca de sus tambos (casas) llamados chagras y es una forma rápida de sembrar plantas medicinales y para su alimentación, claro está, que también un poco alejados de la comunidad en pequeñas parcelas también se realizan cultivos pero a mayor escala y casi siempre incluyen una o dos especias.

Rituales: Los rituales son exclusivos del Jaibaná que es una figura importante dentro de la comunidad con poderes espirituales, sanativos y de protección, en algunos casos es nombrado Gobernador.

\section{Ruta (RAP) Etnoeducativa}




\begin{tabular}{|l|l|}
\hline Momentos & \multicolumn{1}{|c|}{ Ruta vivencial } \\
\hline \multirow{5}{*}{ 1. Inicial } & $\begin{array}{l}\text { Objetivos de aprendizaje: corresponde al fin o las metas al } \\
\text { que el docente quiere llegar, demostrado por los estudiantes en } \\
\text { términos de alcance de competencias, luego del desarrollo de una } \\
\text { plan, propuesta o talleres entre otros métodos de aprendizaje, los } \\
\text { que tienen una didáctica planificada en la unidad. }\end{array}$ \\
\cline { 2 - 2 } & $\begin{array}{l}\text { Temas: son los contenidos de un plan, propuesta o talleres entre } \\
\text { otros de una manera estructurada y organizada de tal forma que } \\
\text { responda a la ruta de aprendizaje que el docente ha desarrollado } \\
\text { para el curso y que está relacionado con los objetivos de } \\
\text { aprendizaje. }\end{array}$ \\
\cline { 2 - 3 } & $\begin{array}{l}\text { Motivación: ubicar la relación entre la sociedad y lo ambiental } \\
\text { para que los estudiantes perciban que hacen parte importante y } \\
\text { activa del medio ambiente. } \\
\text { Dependiendo de las temáticas a desarrollar abordar se plantean } \\
\text { actividades que permitan crear esas reflexiones y motivaciones } \\
\text { personales. }\end{array}$ \\
\cline { 2 - 3 } & $\begin{array}{l}\text { Pensarse y pensarnos (Preconceptos): A partir de la motivación, } \\
\text { preguntaremos a los estudiantes sobre los saberes previos de } \\
\text { la temática pero girando en relación con el reconocimiento del } \\
\text { medio ambiente. }\end{array}$ \\
\hline
\end{tabular}

\begin{tabular}{|c|c|}
\hline \multirow{4}{*}{ 2. Desarrollo } & $\begin{array}{l}\text { Diálogo de saberes (Explicación): Contextualizar los temas (en } \\
\text { el escenario indígena y el no indígena) que se estén tratando. } \\
\text { Identificar en la realidad medio ambiental las diferentes prácticas } \\
\text { y sus impactos en la vida de las personas y sus entornos tanto } \\
\text { indígenas como no indígenas. }\end{array}$ \\
\hline & $\begin{array}{l}\text { Video: Como práctica de utilización de las TIC se plantea la } \\
\text { presentación de un video en relación a los temas que se están } \\
\text { tratando. }\end{array}$ \\
\hline & $\begin{array}{l}\text { Producción intelectual: Después de la explicación y la } \\
\text { observación del video se propone un trabajo grupal donde } \\
\text { reflexionarán teniendo en cuenta unas preguntas preestablecidas } \\
\text { de la temática al final se discute en una plenaria. }\end{array}$ \\
\hline & $\begin{array}{l}\text { Indagación: Proponer una actividad de indagación, donde } \\
\text { el estudiante favorezca su capacidad de investigación y los } \\
\text { procesos que esto implica haciendo uso de distintas técnicas } \\
\text { como la entrevista, la encuesta, la observación entre otros, lo que } \\
\text { al final lleva al estudiante a organizar datos para ser interpretados } \\
\text { y analizados, posterior son socializados. }\end{array}$ \\
\hline
\end{tabular}




\begin{tabular}{|c|c|}
\hline \multirow[b]{2}{*}{ 3. Final } & $\begin{array}{l}\text { Transformando realidades: Colocar en relación cada temática } \\
\text { con su utilidad práctica: analizar, junto con los alumnos, el poder } \\
\text { que tienen de transformar la realidad ambientales, por último, } \\
\text { el estudiante debe reflexionar sobre los temas tratados siempre } \\
\text { relacionándolos con el entorno natural. }\end{array}$ \\
\hline & $\begin{array}{l}\text { Reconstruyendo saberes (Diálogo con las ciencias naturales): } \\
\text { De acuerdo a la experiencia y vivencias se establece una relación } \\
\text { con las ciencias naturales y se propone acciones y prácticas } \\
\text { ambientales sostenibles que se pueda agregar a la cotidianidad } \\
\text { de la escuela, comunidades y familia, como expresión consciente } \\
\text { del aporte y la realidades ancestrales de la etnia Emberá Katío. }\end{array}$ \\
\hline \multicolumn{2}{|r|}{ TALLERES } \\
\hline & Taller 1. Territorio \\
\hline
\end{tabular}

\section{Objetivos de aprendizaje:}

- Comprender que en un ecosistema los seres vivos interactúan con otros organismos y con el ambiente físico, y que los seres vivos dependen de estas relaciones.

- Reconoce características ambientales del entorno y peligros que lo amenazan.

Temas: ecosistema, hábitat, medio ambiente.

Motivación: Introduce al tema con el siguiente comentario: Toda la sociedad humana, para vivir, transforman el espacio natural, por ejemplo, la etnia Emberá Katío considera su espacio natural o medio ambiente como TERRITORIO el cual fue dado por Dios al que ellos llaman (Caragabi), por lo que transforman el espacio en sagrado, y toman todo de él. En este proceso se usan los elementos naturales para adecuar el entorno y alcanzar ciertas condiciones a partir de las cuales las comunidades consideran que pueden vivir bien.

Preconceptos: por medio de preguntas dirigidas se descubren los conocimientos previos que tienen los estudiantes: ¿Cuál crees que es el concepto de ecosistema, hábitat y medio ambiente? ¿Qué puedes aprovechar en los ecosistemas? ¿Cuántas clases de ecosistemas crees que hay? ¿Qué peligros amenazan los ecosistemas?Con las respuestas el docente realiza una síntesis en el tablero utilizando una herramienta cognitiva como: mapas conceptuales, mapas mentales, cuadros sinópticos, entre otros

Explicación: Se explica primero enmarcados en los saberes ancestrales expuestos en la perspectiva de esta propuesta contextualizando con los conceptos de ecosistema, hábitat, tipos de ecosistema, componentes del ecosistema, medio ambiente, problemas ambientales, entre otros aspectos relacionados.

Video: complementa la explicación invitando a los estudiantes a observar el video Medio ambiente y ecosistemas (https://www.youtube.com/watch?v=XtzYwD0dgbQ) 
Producción intelectual: Según lo aprendido pide a los estudiantes que realicen un cuadro comparativo entre un ecosistema urbano y rural, un ecosistema terrestre y otro acuático, que lo complementen con dibujos e identifiquen los componentes bióticos y abióticos, también que identifiquen problemas ambientales ocasionados por el hombre y posibles soluciones.

Indagación: El docente solicita a los estudiantes que procedan a observar los diferentes entornos en los que vive: sus hogares, el barrio o comunidad y el colegio. Tiene que analizar las relaciones que hay con los componentes de la naturaleza, con los animales, las plantas, el agua, el aire, el sol.

Guía de observación:

Hogares: Describe los espacios del hogar en los que existan elementos de la naturaleza: ¿Cómo te relacionas con ellos? ¿Quién de tu hogar los cuida y cómo lo hace? ¿Tú participas en esto? ¿Cómo entran los elementos de la naturaleza al hogar? ¿Qué piensas de esto? ¿Qué sientes cuando te bañas, cuando tomas agua, cuando ves el sol, cuando ves una planta?

El barrio o comunidad zona urbana: escoge un lugar del barrio en el que puedas observar la interacción de la ciudad, los habitantes con los elementos de la naturaleza: puede ser un parque, un río, una quebrada, una construcción, un potrero, etc. ¿Cómo la ciudad (las calles, las construcciones, el cemento, los carros) interactúa con estos elementos naturales? ¿Cómo lo hacen las personas? ¿Cómo lo haces tú? ¿Qué prácticas de cuidado observas que tienen las personas? o, por el contrario, ¿cómo las personas afectan con sus acciones estos elementos? ¿Qué piensas de lo que ves?

El barrio o comunidad zona rural: escoge un lugar del barrio en el que puedas observar la interacción de los habitantes con los elementos de la naturaleza: puede ser un parque, un río, una quebrada, un potrero, etc. ¿Cómo la comunidad (las calles, las viviendas, medios de transporte) interactúa con estos elementos naturales? ¿Cómo lo hacen las personas? ¿Cómo lo haces tú? ¿Qué prácticas de cuidado observas que tienen las personas? o, por el contrario, ¿cómo las personas afectan con sus acciones estos elementos? ¿Qué piensas de lo que ves?

La escuela: observa los lugares que frecuentas, tu salón de clase: ¿Dónde están ubicadas las zonas verdes? ¿Cómo se relacionan tus compañeros con las plantas, con los árboles, con el agua, con el sol, con el clima? ¿Cómo lo haces tú? ¿Qué elementos son los que hacen que exista una relación poco armónica con la naturaleza?

Puedes anexar fotos de lo observado o representarlo mediante imágenes o dibujos para que te sirva en la socialización. 
Transformando realidades: A partir de los resultados de la observación, la explicación y producción intelectual se puede proponer a los estudiantes que desarrollen un proyecto que se pueda llevar a cabo en el colegio, para armonizar la relación entre la comunidad educativa y el cuidado del medio ambiente con el propósito de identificar y transformar problemáticas contribuyendo a la relación armoniosa con el medio ambiente.

Diálogo con las ciencias naturales: Según los problemas identificados y los proyectos o propuestas diseñadas, se les indica a los estudiantes que consulten sobre el impacto de la actividad del hombre en el medio ambiente o en los ecosistemas del contexto, por ejemplo la hidroeléctrica de URRA, entre otros, de igual forma sobre malas prácticas humanas a nivel biológico, químicos y de sostenibilidad ambiental. También, podemos enriquecer lo aprendido con salidas pedagógicas, caminatas ecológicas, que faciliten comprobar de primera mano los distintos problemas ambientales e identificar tipos de ecosistemas y sus componentes y aplicar las competencias ambientales desarrolladas.

\section{Objetivos de aprendizaje:}

- Identificar plantas medicinales de su entorno y su utilización ancestral, clasificándolas usando gráficos, tablas y otras representaciones.

- Proponer acciones de cuidado a las plantas, teniendo en cuenta características como medicinales y relación con el entorno

Temas: La salud, Plantas medicinales, cuidado y preservación de la flora

Motivación: introduce a los alumnos en el tema hablándoles de que en las plantas se acumula el conocimiento ancestral, o sea de las generaciones que las han observado, cuidado, cultivado, procesado y transformado en alimentos y medicinas.

Preconceptos: por medio de preguntas dirigidas se descubren los conocimientos previos que tienen los estudiantes: ¿Qué plantas medicinales conoces y cómo las usas, en qué enfermedades?- con las respuestas se construye una lista de plantas ancestrales utilizadas para la medicina

Explicación: Se explica primero enmarcados en los saberes ancestrales expuestos en la perspectiva de esta propuesta, luego se hace algunas comparaciones con las plantas medicinales de la región no indígena en relación a su obtención, cultivo, preparación, funciones, entre otros aspectos. Se procede a darles las siguientes preguntas: ¿Cuáles son las causas de que no se utilicen con mucha frecuencia las plantas medicinales hoy día? ¿Cómo se puede recuperar los saberes ancestrales sobre las plantas medicinales? con el fin de ser abordadas después de ver el video a continuación.

Video: Se les proyecta un documental: Cómo Cultivar, Transformar y aprovechar Plantas Medicinales https://www.youtube.com/watch?v=Cwe-akaYnK0 
Producción intelectual: después de observar el documental se abre un espacio de producción intelectual que gira alrededor de las preguntas planteadas, para ello, se da un tiempo prudente de discusión grupal, posterior en plenaria se socializan las reflexiones.

Indagación: Cada estudiante deberá indagar con sus familiares, vecinos o allegados, en especial los más ancianos o con conocimiento de plantas, cuáles eran sus prácticas con plantas medicinales, en este sentido previamente se programa con los estudiantes el diseño de una entrevista las preguntas pueden ser las siguientes o pueden cambiar según acuerdos:

1. ¿Menciona las plantas medicinales que ancestralmente utilizan y que enfermedades trataban?

2. ¿En la actualidad todavía son usadas y donde se consiguen?

3. ¿Cómo se cultivan las plantas medicinales?

4. ¿Cómo se preparaba un remedio y como se debe tomar?

5. ¿Cómo se identifican las plantas medicinales de las que no lo son?

6. ¿De dónde obtuvo los conocimientos de las plantas medicinales?

La entrevista les permitirá:

- Identificar plantas medicinales de su entorno y su utilización ancestral, clasificándolas usando gráficos, tablas y otras representaciones.

- Reconocer remedios naturales y tratamientos ancestrales en su familia.

Esta actividad vincula a la familia en el proceso de enseñanza-aprendizaje de sus hijos.

Debe explicar a los estudiantes como recoger la información y si así lo desea diseñar un formato.

Transformando realidades: Con los datos obtenidos en la entrevista los estudiantes socializarán en mesa redonda haciendo uso de diapositivas, videos o carteleras, también se puede invitar a alguna de las personas entrevistadas para que compartan de primera mano el conocimiento. Durante el evento el docente debe encontrar puntos de encuentro entre los saberes ancestrales indígenas y los tradicionales de los no indígenas. Con esta actividad se pretende identificar prácticas comunes, de esta forma los niños y niñas indígenas se sentirán incluidos en el proceso. También puede salir un proyecto de cultivo de plantas medicinales donde pueda participar toda la comunidad educativa incluido los ancianos que participaron en la entrevista. 
Diálogo con las ciencias naturales: Para finalizar, el docente fomenta un diálogo entre los saberes expuestos y las Ciencias Naturales y Educación Ambiental. Permitiendo una reconstrucción de saberes ancestrales en la que los hallazgos evidenciados en la cotidianidad de los estudiantes y sus familias entren en diálogo con las ciencias naturales y a partir de ahí emerjan nuevos conocimientos tanto para los estudiantes como para el docente.

\section{Taller 3. Alimentación}

\section{Objetivos de aprendizaje:}

- Identificar distintas clases de alimentos tradicionales, el origen, materia prima y proceso de su preparación.

- Asociar el cuidado de sus sistemas con una alimentación e higiene adecuadas

- Explicar el camino que siguen los alimentos en el organismo y los cambios que sufren durante el proceso de digestión desde que son ingeridos hasta que los nutrientes llegan a las células.

Temas: La nutrición.

Motivación: Introduce a los estudiantes informándoles que las prácticas alimenticias están relacionadas estrechamente entre las comunidades humanas y el entorno natural en que viven. Las plantas y algunos animales alimentan al cuerpo, para que permanezca sano, fuerte, y poder desarrollarse, pero también alimentan el espíritu de las personas y el saber de las comunidades, porque en ellas se acumula el conocimiento de múltiples generaciones que se han encargado de ir transformando en alimento todos estos recursos. Sin embargo, en el mundo cambiante hoy día estos saberes han ido cambiando y algunos desapareciendo.

Preconceptos: por medio de preguntas dirigidas se descubren los conocimientos previos que tienen los estudiantes: ¿Qué alimentos tradicionales conoces y que ingredientes lleva? ¿Cómo obtienen los productos vegetales para los alimentos? ¿Cómo obtienen los productos animales para los alimentos?- con las respuestas se construye una lista de alimentos ancestrales y los ingredientes que llevan.

Explicación: Se explica primero enmarcados en los saberes ancestrales expuestos en la perspectiva de esta propuesta, luego se hace algunas comparaciones con los alimentos tradicionales indígenas y no indígena en relación a su obtención, cultivo, preparación, funciones, entre otros aspectos. Se procede a darles las siguientes preguntas: ¿Por qué algunos alimentos ancestrales hoy día han desaparecido? ¿Cómo se puede recuperar los saberes ancestrales sobre la alimentación? con el fin de ser abordadas después de ver el video a continuación.

Video: se les muestra un video sobre un documental: Gastronomía Indígena Herencias https://www.youtube.com/watch?v=XId32KAjtZQ

Producción intelectual: después de observar el documental se abre un espacio de producción intelectual que gira alrededor de las preguntas planteadas, para ello, se da un tiempo prudente de discusión grupal, posterior en plenaria se socializan las reflexiones. 
Indagación: Cada estudiante deberá indagar con sus familiares, vecinos o allegados, en especial los más ancianos o con conocimiento de plantas y animales que hacen parte de la alimentación ancestral, cuáles eran sus prácticas con plantas y animales comestibles, en este sentido previamente se programa con los estudiantes el diseño de una entrevista las preguntas pueden ser las siguientes o pueden cambiar según acuerdos:

1. ¿Menciona los alimentos que ancestralmente hacían parte de la gastronomía?

2. ¿Cuál era sus ingredientes?

3. ¿En la actualidad cuáles se preparan?

4. ¿Cómo se cultivan las plantas de donde se obtiene alimento?

5. ¿Cómo se conseguían los animales?

6. ¿Cómo se identifican las plantas comestibles?

7. ¿denos un ejemplo de comidas ancestrales y su preparación?

La entrevista les permitirá:

- Identificar distintas clases de alimentos tradicionales, el origen, materia prima y proceso de su preparación.

- Reconocer comidas ancestrales en su familia.

Esta actividad vincula a la familia en el proceso de enseñanza-aprendizaje de sus hijos.

Debe explicar a los estudiantes como recoger la información y si así lo desea diseñar un formato.

Transformando realidades: Con los datos obtenidos en la entrevista los estudiantes socializarán en mesa redonda haciendo uso de diapositivas, videos o carteleras, también se puede invitar alguna de las personas entrevistadas para que compartan de primera mano el conocimiento. Durante el evento el docente debe encontrar puntos de encuentro entre los saberes ancestrales indígenas y los tradicionales de los no indígenas. Con esta actividad se pretende identificar prácticas comunes, de esta forma los niños y niñas indígenas se sentirán incluidos en el proceso. También puede salir un proyecto de emprendimiento en relación a la conformación de una empresa de dulces tradicionales o galletas, entre otros productos donde pueda participar toda la comunidad educativa incluido los ancianos que participaron en la entrevista. 
Diálogo con las ciencias naturales: Para finalizar, el docente fomenta un diálogo entre los saberes expuestos y las Ciencias Naturales y Educación Ambiental. Permitiendo un reconstrucción de saberes ancestrales en la que los hallazgos evidenciados en la cotidianidad de los estudiantes y sus familias entren en diálogo con las ciencias naturales y a partir de ahí emerjan nuevos conocimientos tanto para los estudiantes como para el docente, también con el fin de lograr los objetivos de aprendizaje: asociar el cuidado de sus sistemas con una alimentación e higiene adecuadas y explicar el camino que siguen los alimentos en el organismo y los cambios que sufren durante el proceso de digestión desde que son ingeridos hasta que los nutrientes llegan a las células.

\section{Taller 4. Chagra}

Objetivos de aprendizaje:

- Comprender el proceso de crecimiento y desarrollo de los seres vivos (las plantas del huerto escolar).

- Utilizar procedimientos ancestrales para el cultivo de productos de la región.

- Reconoce algunas actividades humanas que generan impactos ambientales positivos y negativos.

Temas: Las plantas, huerto escolar.

Motivación: Contextualizar la relación entre los saberes ancestrales indígenas y lo ambiental de modo que los estudiantes generen reflexiones y motivaciones personales con la temática a tratar. Razón por la cual se propone la presentación de un video: Embera Katíos - Un pueblo indígena con historia y cultura propia (https://www.youtube.com/watch?v=Y69S0MRS3N8) y formulamos preguntas generadoras relacionadas con los objetivos de aprendizaje. ¿Cómo cultivan las comunidades indígenas? ¿Qué características observas del medio ambiente donde viven los indígenas y como se diferencia del urbano dónde estamos?

Preconceptos: por medio de preguntas dirigidas se descubren los conocimientos previos que tienen los estudiantes: ¿menciona los cuidados que hay que tener con las plantas? ¿Qué tipo de plantas tienes en tu casa y como son las prácticas de sembrado? ¿Por qué crees es bueno tener plantas en casa? ¿Menciona algunas plantas de donde provienen alimentos que tú consumes en tu casa? ¿Menciona como son las prácticas de cultivo que práctican en tu casa o en la comunidad? Con las respuestas el docente realiza una síntesis en el tablero utilizando una herramienta cognitiva como: mapas conceptuales, mapas mentales, cuadros sinópticos, entre otros. 
Explicación: Para desarrollar el tema inicialmente se expone un video sobre la huerta escolar: Del Huerto Escolar a la Alimentación y Salud Escolar (https://www. youtube.com/watch?v=MUZhTq7ApOs) ¿en el video como están construido el huerto escolar? ¿Qué plantas alcanzas a distinguir en el huerto escolar? ¿Por qué los niños del video utilizan materiales reciclables en el huerto escolar? ¿Para qué se utilizan los productos recolectados en el huerto escolar? ¿Menciona los beneficios a nivel ambiental y biológico de la implementación de un huerto escolar? ¿Quiénes participan en el huerto escolar? Seguido el docente hace una comparación con las Chagras que son pequeños cultivos indígenas. Posterior se hace el abordaje temático, profundizando en los procesos de fotosíntesis, germinación, de siembra, cultivo, los cuidados que requiere la planta. También se describe sobre la utilización e importancia de las chagras en la comunidad Emberá.

Producción intelectual: por medio de preguntas se verifica la producción cognitiva: ¿Cómo son las chagras y que productos siembran? ¿Por qué son importantes las chagras en la comunidad Emberá Katío? ¿Qué se necesita para hacer una chagra escolar? ¿Cómo se puede adecuar el espacio para sembrar plantas? ¿Qué plantas se pueden sembrar en la chagra escolar? ¿Cómo sería el cuidado del huerto escolar y cómo se utilizaría? Esta actividad puede ser grupal y posterior se hace una plenaria para su socialización.

Indagación: Cada estudiante deberá indagar con sus familiares, vecinos o allegados, en especial los que realizan prácticas agrícolas sobre las siguientes preguntas: ¿Cómo cree que se puede hacer un huerto escolar? ¿Qué herramientas y materiales se necesitan? ¿Qué plantas se pueden sembrar? ¿Cómo son los cuidados para tener excelentes resultados? ¿Cuáles son los tiempos desde el semillero hasta la recolecta de productos? Posterior a la recolección de los resultados los estudiantes deben diseñar una cartilla con los datos y en lo posible incluir imágenes.

Transformando realidades: El docente propone un proyecto de huerto escolar donde estén implicados los estudiantes y los padres de familia que lo deseen "es un trabajo colaborativo", para ello hay que gestionar con las directivas el espacio, herramientas, materiales entre otros requisitos. Si es exitoso se transforman realidades tanto de los estudiantes como la de sus familias. La elaboración de un huerto escolar que incluye la siembra y el cuidado de las plantas que proporcionan algún beneficio como alimentación y medicinales, trascienden las fronteras del colegio; el éxito depende del trabajo en equipo, de la motivación y creatividad de sus actores directos, de su constancia y persistencia.

Diálogo con las ciencias naturales: Este proyecto busca rescatar las prácticas ancestrales ecológicas y el cuidado de las plantas que las comunidades indígenas y no indígenas según los resultados de las indagaciones en este taller. El propósito es que los estudiantes reconozcan algunas actividades humanas que generan impactos ambientales positivos y negativos, también que propongan nuevas formas de relacionarse con su entorno, creen hábitos de cuidado de la naturaleza y entre ellos mismos; y adquieran un conocimiento teórico y práctico de la biología y el medio ambiente. 


\section{Objetivos de aprendizaje:}

Comprender el impacto ambiental mediante comparaciones historia y presente

Valorar los recursos naturales en torno a su cuidado, conservación y prevención de daños futuros

Temas: Medio ambiente, recursos naturales

Motivación: Por medio de la historia del origen del agua según la etnia Emberá Katío (Árbol Jenené) se introduce en el tema y se reflexiona sobre la importancia del agua para todos los seres vivos.

Preconceptos: por medio de preguntas dirigidas se descubren los conocimientos previos que tienen los estudiantes: ¿según sus creencias cual es el origen del agua? ¿Cómo era el rio Sinú en la antigüedad y como es en el presente? ¿Cómo piensas que el hombre explota los recursos naturales? Con las respuestas el docente realiza una síntesis en el tablero utilizando una herramienta cognitiva como: mapas conceptuales, mapas mentales, cuadros sinópticos, entre otros.

Explicación: Se explica que el medio ambiente guarda muchas memorias (culturales, rituales, religiosas, festividades y su estructura natural) por ejemplo una memoria del medio natural es que ancestralmente se utilizaba los ríos por los indígenas como medio de transporte y fuente de pesca abundante, hoy día esta práctica se ha disminuido por causa de la intervención del hombre cuando construyeron la represa URRA. Otra memoria son las prácticas de bailes, música, comidas, artesanías, maquillaje que también están desapareciendo pero quedan algunos conocedores que pueden transportarnos a esas memorias y rescatar algunas prácticas ancestrales.

Video: se les muestra un video: Documental wounaan taller memoria ancestral (https://www.youtube.com/watch?v=uUhd2egpSV0)

Producción intelectual: por medio de preguntas se verifica la producción cognitiva: ¿Cómo es el medio ambiente antes en las comunidades indígenas y como es hoy día? ¿Cómo son aprovechados los recursos naturales (agua, tierra, plantas, animales) en la antigüedad y en el presente? ¿Cómo crees se pueden rescatar algunas memorias de buenas prácticas ambientales? Esta actividad puede ser grupal y posterior se hace una plenaria para su socialización.

Indagación: Cada estudiante deberá indagar con sus familiares, vecinos o allegados sobre las siguientes preguntas: ¿mencionar algunas memorias ancestrales de ritos, leyendas, cultura, alimentación, artesanías, maquillajes, y cambios medioambientales? ¿Hoy día cuáles de esas memorias han tenido cambios? ¿Por qué cree que se dieron esos cambios? Posterior a la recolección de los resultados los estudiantes deben socializar los resultados ante los compañeros. 
Transformando realidades: En un trabajo grupal los estudiantes organizan una feria "rescatando memorias" donde hacen muestras gastronómicas, culturales, ritos, leyendas, explotación de recursos, entre otras memorias ancestrales importantes, el docente los organiza y lo guía en el proyecto, donde se invita a la comunidad educativa para visitar las diferentes exposiciones. Se busca rescatar prácticas como la pintura corporal de los indígenas, bailes, instrumentos, artesanías y comparar estas prácticas con las del día de hoy.

Diálogo con las ciencias naturales: Este proyecto busca rescatar memorias ancestrales ecológicas, culturales, religiosas y sociales que las comunidades indígenas y no indígenas presentan según los resultados de las indagaciones en este taller. El propósito es que los estudiantes reconozcan algunas memorias que visibilicen los cambios ambientales en el tiempo ya sean positivas o negativas, también que propongan nuevas formas de relacionarse con su entorno, creen hábitos de cuidado de la naturaleza y entre ellos mismos; y adquieran un conocimiento teórico y práctico de la biología y el medio ambiente.

\begin{tabular}{|l|l|l|}
\multicolumn{4}{|c|}{ EJEMPLOS DE DBA QUE SE PUEDEN ENMARCAR EN LOS TALLERES } \\
\hline APRENDIZAJES & \multicolumn{2}{c|}{ EVIDENCIAS } \\
\hline C o m p r e $\mathrm{n} \mathrm{d} \mathrm{e}$ & - & Diferencia tipos de ecosistemas (terrestres y acuáticos) \\
que & existen & correspondientes a distintas ubicaciones geográficas, para \\
distintos & tipos & establecer sus principales características.
\end{tabular}
de ecosistemas (terrestres $y$

- Explica cómo repercuten las características físicas a c u á t i cos o (temperatura, humedad, tipo de suelo, altitud) de y que sus ecosistemas (acuáticos y terrestres) en la supervivencia de características los organismos que allí habitan.

f í s i c a s . Propone representaciones de los ecosistemas (temperatura, representativos de su región, resaltando sus particularidades humedad, tipos de suelo, altitud) permiten que (especies endémicas, potencialidades ecoturísticas, entre otros.) y plantea estrategias para su conservación.

habiten en ellos diferentes seres vivos 


\begin{tabular}{|c|c|}
\hline $\begin{array}{l}\text { Comprende que } \\
\text { en los seres } \\
\text { humanos (y en } \\
\text { muchos otros } \\
\text { animales) la } \\
\text { nutrición involucra } \\
\text { el funcionamiento } \\
\text { integrado de } \\
\text { un conjunto } \\
\text { de sistemas } \\
\text { de órganos: } \\
\text { d i g e s t i v o, } \\
\text { respiratorio y } \\
\text { circulatorio }\end{array}$ & $\begin{array}{l}\text { - Explica el camino que siguen los alimentos en el organismo } \\
\text { y los cambios que sufren durante el proceso de digestión } \\
\text { desde que son ingeridos hasta que los nutrientes llegan a } \\
\text { las células. } \\
\text { - Explica por qué cuando se hace ejercicio físico aumentan } \\
\text { tanto la frecuencia cardíaca como la respiratoria y vincula } \\
\text { la explicación con los procesos de obtención de energía de } \\
\text { las células. }\end{array}$ \\
\hline $\begin{array}{l}\text { Co m p r e n d e } \\
\text { que los seres } \\
\text { vivos (plantas y } \\
\text { animales) tienen } \\
\text { características } \\
\text { comunes (se } \\
\text { a I i m e n t a } \text {, } \\
\text { respiran, tienen } \\
\text { un ciclo de vida, } \\
\text { responden al } \\
\text { entorno) y los } \\
\text { diferencia de los } \\
\text { objetos inertes. }\end{array}$ & $\begin{array}{l}\text { - Clasifica seres vivos (plantas y animales) de su entorno, } \\
\text { según sus características observables (tamaño, cubierta } \\
\text { corporal, cantidad y tipo de miembros, forma de raíz, tallo, } \\
\text { hojas, flores y frutos) y los diferencia de los objetos inertes, } \\
\text { a partir de criterios que tienen que ver con las características } \\
\text { básicas de los seres vivos. } \\
\text { - Compara características y partes de plantas y animales, } \\
\text { utilizando instrumentos simples como la lupa para realizar } \\
\text { observaciones. } \\
\text { - Describe las partes de las plantas (raíz, tallo, hojas, flores } \\
\text { y frutos), así como las de animales de su entorno, según } \\
\text { características observables (tamaño, cubierta corporal, } \\
\text { cantidad y tipo de miembros). }\end{array}$ \\
\hline
\end{tabular}

Nota. Fuente: elaboración propia (2020)

\section{CONCLUSIONES}

El trabajo cualitativo, comprensivo, interpretativo, etnográfico, realizado por los investigadores con apoyo de la comunidad y referentes visibles de la etnia Emberá Katio, permitió el diseño de una propuesta con cinco talleres que hacen énfasis en las categorías emergentes mencionadas, brindando una ruta metodológica inclusiva e intercultural. Empero, es preciso que antes de llegar a una aplicación, el docente se prepare en relación a la etnoeducación en ambientes interculturales, en concordancia al hablar de diálogo de saberes en la escuela intercultural, no solo se orienta hacia la diversidad de la cultura de estudiantes, también apunta a la diversidad cultural de los docentes, a los diferentes miembros de la comunidad educativa, a los aprendizajes, a la evaluación, en general a todo lo que compone el currículo, y todos ellos son quienes dialogarían eventualmente (Arias, 2018).

También, hay que resaltar otras connotaciones como las del grupo de etnógrafos, cuyas implicaciones fueron significativas por la inmersión directa en la comunidad Doza Emberá Katío, logrando una empatía, una aproximación 
a las experiencias, hechos y saberes ancestrales que terminaron en un análisis riguroso. Seguido se desarrollaron reflexiones las que permitieron la construcción de una propuesta desde la etnoeducación con bases en categorías emergentes, dando respuesta a la pregunta de investigación. Fue una práctica enriquecedora y rigurosa con validez científica que se presenta a la comunidad académica regional, nacional e internacional.

En cuanto a los beneficios de esta investigación se da en los siguientes componentes: En los estudiantes, en contextos interculturales el presente estudio promueve la integración de saberes ancestrales sobre el medio ambiente al plan de área de Ciencias Naturales y Educación Ambiental con el fin de desarrollar procesos etnoeducativos donde los estudiantes indígenas y no indígenas se sientan implicados en la práctica, exista un trabajo en equipo, y se logre ese diálogo entre las Ciencias Naturales y las Cosmovisiones Indígenas acerca de lo ambiental. Todo esto, con el firme propósito de generar condiciones pedagógicas para que los y las estudiantes se motiven en el acto de aprehender, recrear y crear conocimientos (Peña, 2016).

En la escuela, su principal beneficio es en la integración de la propuesta a su currículo, teniendo presente que se diseñó incluyendo talleres didáctico/pedagógicos en ambientes etnoeducativos interculturales de Ciencias Naturales y Educación Ambiental, soportada con la sistematización de saberes ancestrales y un método de Reflexión - Acción - Participación (RAP) para el desarrollo de la estrategia que apuntan a transformar la escuela en un espacio de formación intercultural, por lo menos en lo que se refiere a las clases de Ciencias Naturales.

En lo social, este tipo de estrategias interculturales permite que los y las estudiantes estrechen nexos de armonía, amistad y promueve el trabajo en grupo, también se ven inmiscuidos los padres de familia y/o acudientes, y sobra decir los docentes y las directivas. Lo que propicia el desarrollo de competencias sociales, ciudadanas y favorece la inteligencia emocional

\section{REFERENCIAS BIBLIOGRÁFICAS}

Aceros Hernández, D. (2019). Problemáticas alrededor del reconocimiento de la comunidad Emberá Katío como sujeto de derecho: el caso del desplazamiento por la construcción de la represa de Urrá. (Tesis de pregrado). Pontificia Universidad Javeriana. Bogotá. Recuperado de http://hdl.handle.net/10554/40913

Aguado, A., \& Campo, Á. (2018). Desarrollo de competencias científicas en biología con la metodología del aprendizaje basado en problemas en estudiantes de noveno grado. Revista Bio-grafía, 11(20), 67-78.

Alcaldía de Tierralta. (2019). Plan de Desarrollo 2016-2019.

Arias, F. (2018). El Diálogo de Saberes entre las Ciencias Naturales y las Cosmovisiones Indígenas acerca de la naturaleza: Una perspectiva para la educación intercultural en el Guaviare. [Tesis doctoral, Universidad Nacional de Colombia-Sede Bogotá]. http://bdigital.unal. edu.co/70182/1/tesis_Frank_\%20Edisson_\%20 Arias.pdf

Ausubel, D. (1998). Teoría del aprendizaje significativo. Fascículos de CEIF, 1, 1-10. http://files.gersain.webnode.es/2000000267721a781a1/Aprendizaje_significativo.pdf

Bruner, J. (1961). Aprendizaje por descubrimiento. NYE U: Iberia.

Castillo, L. (2015). Conflictos ambientales y movimientos sociales: el caso del movimiento embera katío en respuesta a la construcción de la represa Urrá (1994-2008). Memoria y sociedad, 19(39), 94-105. https://revistas. 
javeriana.edu.co/index.php/memoysociedad/ article/download/13404/10778

DANE (2019). Población Indígena de Colombia. Resultados del censo nacional de población y vivienda 2018. Recuperado de https://www.dane. gov.co/files/investigaciones/boletines/gruposetnicos/presentacion-grupos-etnicos-2019.pdf

Fresneda Páez, M. A. (2019). Evaluación y modelo pedagógico en el colegio.

González, A. (2010). Educación para la paz y la convivencia en la escuela. Publicación del Instituto para la Investigación Educativa y el Desarrollo Pedagógico, Bogotá D.C. Enero de 2010. No. 75

Hernández Sampieri, R., Fernández Collado, C., \& Baptista Lucio, P. (2014). Metodología de la investigación. Sexta Edición. México: Editorial Mc Graw Hill

Leymonié, J. (2009). Aportes para la enseñanza de las Ciencias Naturales. Segundo Estudio Regional Comparativo y Explicativo. Organización de las Naciones Unidas para la Educación la Ciencia y la Cultura. Oficina Regional de Educación para América Latina y el Caribe. Santiago, Chile.

Medina, M. A. G., \& Cantero, C. L. (2018). Interculturalidad de las Etnias en Colombia.

Melo, N. B. (2019). Enseñanza a partir de saberes tradicionales de las comunidades de la etnia wayuu. Educación y educadores, 22(2), 237255. https://educacionyeducadores.unisabana. edu.co/index.php/eye/article/view/10198/5575

Muñoz Garzón, Z. (2018). La ciudadanía ambiental una apuesta de construcción colectiva. [Trabajo de maestría, Universidad de Ciencias Aplicadas y Ambientales] https://repository.udca. edu.co/jspui/bitstream/11158/995/1/TESIS $\% 20$ MAESTRIA\%20UDCA.pdf
Muñoz Rojas, T. M., Giraldo Builes, J., \& López Gómez, M. del S. (2019). Mecanismos de protección de los conocimientos tradicionales: el caso de Colombia. Revista Derecho Del Estado, (43), 235-264. Recuperado de https:// doi.org/10.18601/01229893.n43.09

Pozo, J. I. y Gómez, M. A. (1998). Aprender y enseñar ciencia. Del conocimiento cotidiano al conocimiento científico. (Ed. Morata, Madrid).

Peña Malagón, E. O. (2016). La RAP, estrategia para la enseñanza de los derechos humanos y el territorio. [Trabajo de maestría, Universidad Pedagógica] http://repository.pedagogica.edu. $\mathrm{co} / \mathrm{bitstream} / \mathrm{handle} / 20.500 .12209 / 3135 / T E-$ 19702.pdf?sequence $=1$

Pérez Caballero, A. J., \& Mendoza, S. (2018). La inclusión de los saberes ancestrales al currículo de la Institución Educativa Técnica Agropecuaria Benkos Bioho. [Disertación doctoral, Universidad de Cartagena]. http://repositorio.unicartagena. edu.co/bitstream/handle/11227/9336/PerezA. pdf?sequence $=1$

Quilaqueo R., Sartorello. \& Torres C. (2020). Diálogo de Saberes en Educación Intercultural en Educación Intercultural: Conflicto Epistémico en Contextos Indígenas de Chile y México. Centro de Investigación en Educación en Contexto Indígena e Intercultural / CIECII, Universidad Católica de Temuco, 2020, 224 p. https://inide.ibero.mx/assets_front/assets/ libros/2020/diálogo2020.pdf

SED de Bogotá, A. M. (2014). Currículo para la excelencia académica y la formación integral. Orientaciones para el área de Ciencias Naturales. Alcaldía de Bogotá. http://www. colombiaaprende. edu. co/html/micrositios/1752/ articles-349445_bogociencias_pdf. pdf.

Soler, M. G., Cárdenas, F. A., \& HernándezPina, F. (2018). Enfoques de enseñanza y 
enfoques de aprendizaje: perspectivas teóricas promisorias para el desarrollo de investigaciones en educación en ciencias. Ciência \& Educação (Bauru), 24(4), 993-1012. https:// www.scielo.br/scielo.php?pid=S151673132018000400993\&script=sci_arttext

Solís Morán, S. M. (2019). Estrategias de enseñanza etnoeducativas en la recuperación de la identidad cultural del pueblo afro ecuatoriano en la unidad educativa "Valle del Chota". [Trabajo de grado, Universidad Central del Ecuador]

Vallejo, F. S., \& Majore, I. A. D. (2018). Los embera katio del Alto Sinú-Córdoba. Oraloteca, (9), 64-84. http://revistas.unimagdalena.edu.co/ index.php/oraloteca/article/view/2901 